cooling factor - might it have played a role in causing the little ice age?

The debate continues among climatologists, but it would seem that Ruddiman must be at least partly correct: there can be no question that Stone Age farmers added methane and carbon dioxide to the atmosphere, but how much and when? The debate, which seems to centre on the interpretation of details of temperature inferences from isotopic records in sediments and ice cores, cannot disguise the fact that levels of both methane and carbon dioxide began to increase long before the onset of industrialization.

Robert J. Charlsonis in the Department of Atmospheric Sciences and the Department of Chemistry, University of Washington, Seattle, Washington 98125, USA.

\title{
Sticking with nature
}

\section{The Gecko's Foot. Bio-inspiration: Engineered from Nature. by Peter Forbes \\ Fourth Estate: 2005.272 pp. $€ 20$}

\section{R. McNeill Alexander}

Biomimetics is the application of ideas from nature in engineering. At first sight, it seems a promising approach: evolution by natural selection is extremely effective, and designers can surely learn from its solutions. But so far, biomimetics has not achieved very much. As recently as 2003, Julian Vincent, professor of biomimetics at the University of Bath, UK, and one of its keenest proponents, wrote that "the only successful examples I know of are Velcro (1955) and the Anglepoise lamp".

I would discount the Anglepoise lamp. Its springs will support it in any position, much as the elastic ligament in a cow's neck supports its head, but I know of no evidence that the lamp designer knew about the cow. Velcro is more convincing. Its inventor, George de Mestral, got the idea from burs that stuck to his dog's

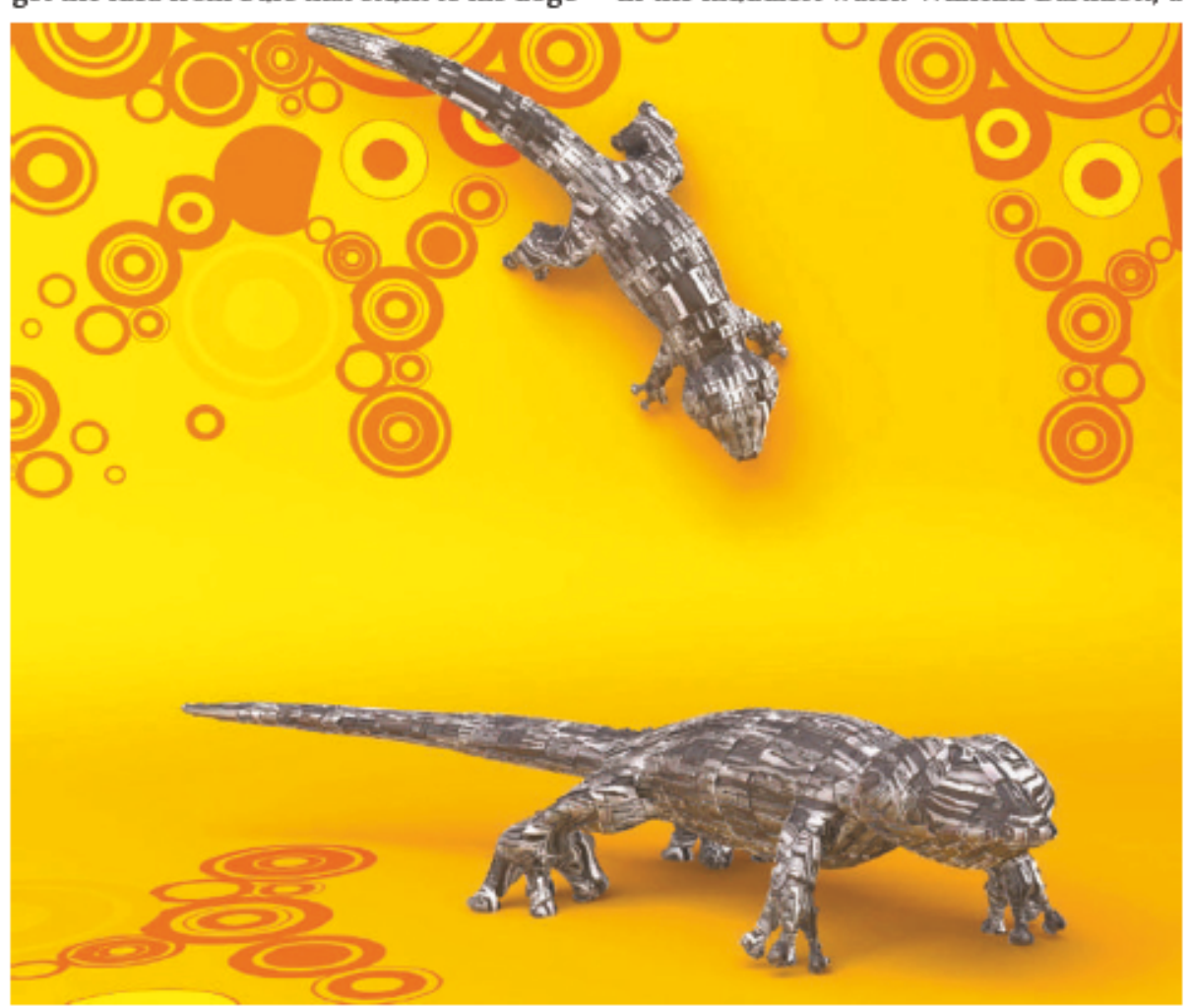

botanist at the University of Bonn, Germany, discovered that these leaves are covered in tiny hydrophobic tubercles, in the blind-zone range of sizes. On this surface, water forms almost spherical drops, and rolls off as if from a duck's back, carrying dirt away with it. (The duck's back works on the same principle.) Barthlott persuaded a paint company that the lotus effect, as he called it, had commercial potential, and they developed a paint for the outside of buildings. It looks like ordinary paint but has a silicone surface that is rough in the blindzone scale. Rain keeps it beautifully clean.

Forbes continues with other examples. The gecko's foot, which gives his book its title, is covered with a carpet-like pile of hairs. The end of each hair has many branches a few hundred nanometres across. These hairs make such close contact with the surfaces on which the lizard walks that they adhere by intermolecular attraction (the van der Waals effect). This lets the gecko climb walls and even run across the ceiling. Adhesive tape working on the gecko principle has been made but not yet commercially developed.

Forbes discusses other examples in the size range that he thinks so promising, including the multilayer reflectors in the wings of iridescent butterflies and the self-assembling properties of bacteriophages but, as with the gecko, these are potential rather than realized commercial applications. He discusses some larger-scale effects, including the aerodynamics of insect flight and the folding pattern that enables a large leaf to be opened from a slender bud, but these are also of possible future value rather than current successes.

Forbes frankly admits that biomimetics has had few triumphs so far. He tells us in his final chapter: "I have written about science and technology on the hoof because this is a new science and success is a matter of decades of work. Rather than wait until some of these technologies have become commonplace, I have tried to capture the Wordworthian 'bliss-it-was-in-that-dawn-to-be-alive' moment of seeing what was, until 15 years ago, a wholly unexpected science taking shape before our eyes."

There is a great deal of interesting and unfamiliar material here, but I found some of the scientific explanations unsatisfactory. To quote one example, in the explanation of the lotus effect, the statement that "water sits on the points of the bumps, with the compression of the air in the cavities giving extrabuoyancy" is misleading: the effect depends on contact angles, not air pressure and buoyancy. On the other hand, an admirably comprehensive set of notes directs readers to the primary scientific literature. The general readers for whom this book is intended will enjoy it, and it will give them some appreciation of a fascinating field.

R. McNeill Alexander is in the Faculty of Biological Sciences, Miall Building, University of Leeds, Leeds LS2 9JT, UK. 